\title{
ACADEMIA NACIONAL DE SEGURIDAD PÚBLICA
}

\section{INFORME DE RENDICIÓN DE CUENTAS}

\section{DE JUNIO 2012 A JULIO 2013}







\section{ACADEMIA NACIONAL DE SEGURIDAD PÚBLICA}

La Academia Nacional de Seguridad Pública, ANSP, es una institución autónoma, de derecho público, adscrita al Ministerio de Justicia y Seguridad Pública. Entre sus principales atribuciones legales están la formación profesional de los policías, según requerimientos que exija la Policía Nacional Civil; elaborar los planes de selección y realizar las pruebas respectivas para el ingreso a la Academia y la promoción en la Policía Nacional Civil; evaluar sistemáticamente al personal policial y organizar los cursos correspondientes para los efectos de promoción y ascensos de los miembros de la Policía Nacional Civil, en todos los niveles y categorías (Art. 2, Ley Orgánica de la ANSP). De conformidad con esta ley, las autoridades principales de la ANSP son la Dirección General y el Consejo Académico.

\section{Misión Institucional}

Formar integralmente y con excelencia académica a policías capaces de garantizar la seguridad de la población, con respeto a los Derechos Humanos.

\section{Visión Institucional}

Ser una institución de alto prestigio nacional e internacional, reconocida por sus aportes en la investigación científica y por la excelencia de sus graduadas y graduados como policías, que gocen de la confianza de la población.

\section{CUMPLIMIENTO DEL PLAN ESTRATÉGICO INSTITUCIONAL (PEI) 2010-2014}

A continuación se presenta el resumen de cumplimiento de resultados hasta julio de 2013 de las líneas estratégicas del PEI 2010-2014, el cual permite visualizar el avance global en términos porcentuales, así como el tipo de cumplimiento alcanzado.

\begin{tabular}{|l|c|c|c|c|c|}
\hline \multicolumn{1}{|c|}{ Líneas Estratégicas } & $\mathbf{2 0 1 0}$ & $\mathbf{2 0 1 1}$ & $\mathbf{2 0 1 2}$ & $\mathbf{2 0 1 3}$ & $\mathbf{2 0 1 4}$ \\
\hline $\begin{array}{l}\text { LE 1. Formación con excelencia } \\
\text { académica de policias integrales y } \\
\text { al servicio de la comunidad. }\end{array}$ & $98.6 \%$ & $82.9 \%$ & $98.0 \%$ & & \\
\hline $\begin{array}{l}\text { LE 2 Investigación Académica } \\
\text { Relativa a la Policia y a la } \\
\text { Seguridad Pública. }\end{array}$ & $91.0 \%$ & $93.8 \%$ & $91.7 \%$ & & \\
\hline $\begin{array}{l}\text { LE 3 Direccionamiento estratégico } \\
\text { con efectiva coordinación entre la } \\
\text { ANSP y la PNC }\end{array}$ & $100.0 \%$ & $93.8 \%$ & $100.0 \%$ & & \\
\hline $\begin{array}{l}\text { LE 4 Institucionalización de la } \\
\text { Equidad e lgualdad de Género }\end{array}$ & $100.0 \%$ & $100.0 \%$ & $100.0 \%$ & & \\
\hline $\begin{array}{l}\text { LE G Gestión Efectiva y } \\
\text { Transparente de los Recursos }\end{array}$ & $92.0 \%$ & $97.4 \%$ & $96.7 \%$ & & \\
\hline Nivel de cumplimiento & $96.3 \%$ & $96.3 \%$ & $97.3 \%$ & & \\
\hline Categoría & Satisfactorio & Satisfactorio & Satisfactorio & & \\
\hline \multicolumn{1}{|c|}{ Ponderación PEl } & $10 \%$ & $\mathbf{2 0} \%$ & $\mathbf{3 0 \%}$ & $\mathbf{3 0 \%}$ & $\mathbf{1 0 \%}$ \\
\hline
\end{tabular}

\begin{tabular}{|c|c|c|}
\hline $\begin{array}{c}\% \text { del } \\
\text { umbral por } \\
\text { categorias }\end{array}$ & CATEGORIA & SUGERENCIA \\
\hline 100 Y MAS & EXCELENTE & Mejora continua \\
\hline $75-99$ & SATISFACTORIA & Acción de mejora \\
\hline $\mathbf{5 5 - 7 4}$ & BUENO & Acción preventiva \\
\hline $\begin{array}{c}\text { Menos de } \\
55\end{array}$ & DEFICIENTE & Acción correctiva \\
\hline
\end{tabular}

El avance del PEI hasta el año 2012 es del 60\% quedando el 40\% pendiente de ejecutar en los años 2013 y 2014. Los niveles de cumplimiento de cada año, han sido situados en la categoría "satisfactorio", según tabla de rangos por categoría establecida en el Sistema Institucional de Planificación, que es la herramienta informática, a través de la cual se monitorea y se da seguimiento a lo programado en el Plan Anual Operativo (PAO). 


\section{FORMACIÓN CON EXCELENCIA ACADÉMICA DE POLICÍAS INTEGRALES Y AL SERVICIO DE LA COMUNIDAD}

Durante el año 2011 y en concordancia con las metas establecidas en el Plan Operativo Anual, el Consejo Académico inició la revisión para la aprobación de los fundamentos del Sistema Educativo Policial Integral (SEPI), como parte de este proceso se ha capacitado al personal docente en diferentes técnicas didácticas y de planificación curricular; además en el tema de investigación educativa, para proporcionarles instrumentos conceptuales, metodológicos y didácticos para el desarrollo de sus actividades de investigación y proyección social. En marzo de dos mil doce, la División de Estudios presentó al Consejo Académico los documentos para la gestión de la autorización de la ANSP como Institución de Educación Superior, mismos que fueron aprobados para que por medio de la Dirección General, fueran remitidos al señor Ministro de Justicia y Seguridad Pública, las gestiones concernientes a la acreditación de la Academia Nacional de Seguridad Pública como Instituto de Educación Superior están en fase de análisis en Casa Presidencial. Una vez se supere esta etapa se remitirá para su aprobación por el Ministerio de Educación a efectos de validar el Plan de Estudios propuesto y para que se haga efectiva las extensiones de títulos correspondientes a los distintos niveles concebidos en el Sistema Educativo Policial Integral.

\section{GRADUACIONES EN EL PERÍODO COMPRENDIDO DEL 1 DE JUNIO DE 2009 AL 31 DE JULIO DE 2013}

Alumnos graduados del Nivel Básico Categoría de Agente: La administración actual desde el 1 de junio de 2009 al 31 de julio de 2013, ha fortalecido la plantilla policial con un total de 3,813 policías de catorce promociones; de los cuales 697 se formaron en el período comprendido entre el 1 de junio de 2012 al 31 de julio de 2013 y correspondientes a las promociones 106 y 107 del nivel básico; más 571 ex policías de quince cursos de actualización que reingresaron a la Policía Nacional Civil, provenientes de remanentes anteriores; haciendo un total de 4,384 policías. A continuación el detalle en el cuadro siguiente:

\begin{tabular}{|c|c|c|c|c|}
\hline \multicolumn{5}{|c|}{$\begin{array}{l}\text { FORMACIÓN INICIAL EN NIVEL BÁSICO } \\
\text { Graduados P-95 a P-107 de junio } 2009 \text { a julio } 2013\end{array}$} \\
\hline Total de graduados & Mujeres & $\%$ & Hombres & $\%$ \\
\hline 3,813 & 770 & $20 \%$ & 3,043 & $80 \%$ \\
\hline \multicolumn{5}{|c|}{$\begin{array}{c}\text { ACTUALIZACIÓN Y REFORZAMIENTO PARA ASPIRANTES A REINGRESAR A LA PNC } \\
\text { Graduados P-03 (grupos del } 1 \text { al 15) de junio } 2009 \text { a julio } 2013\end{array}$} \\
\hline Total de graduados & Mujeres & $\%$ & Hombres & $\%$ \\
\hline 571 & 39 & 7 & 532 & 93 \\
\hline
\end{tabular}

Cursos de Ascenso en niveles Básico, Ejecutivo y Superior: En el período de junio de 2009 a julio 2013 la actual administración graduó en cursos de ascenso a un total de 1,354 integrantes de la PNC tal como se muestra a continuación: 
Informe de rendición de cuentas ANSP, junio 2012 - julio 2013 $\pi^{000}$

\begin{tabular}{|c|c|c|c|c|c|}
\hline \multicolumn{6}{|c|}{$\begin{array}{c}\text { CURSOS DE ASCENSO EN NIVELES BÁSICO, EJECUTIVO Y SUPERIOR } \\
\text { junio de } 2009 \text { a julio de } 2013\end{array}$} \\
\hline Cursos & Total de graduados & Mujeres & $\%$ & Hombres & $\%$ \\
\hline Ascenso de Agente a Cabo & 489 & 46 & 9 & 443 & 91 \\
\hline Ascenso de Cabo a Sargento & 363 & 18 & 5 & 345 & 95 \\
\hline Consolidación a Cabo & 52 & 3 & 6 & 49 & 94 \\
\hline Consolidación a Sargento & 25 & 2 & 8 & 23 & 92 \\
\hline Ascenso de Subinspector a Inspector & 253 & 24 & 9 & 229 & 91 \\
\hline Ascenso de Inspector a Inspector Jefe & 33 & 5 & 15 & 28 & 85 \\
\hline Ascenso de Sargento a Subinspector & 33 & 1 & 1 & 32 & 99 \\
\hline Curso a Subinspector & 41 & 1 & 2 & 40 & 98 \\
\hline Ascenso de Inspector Jefe a Subcomisionado & 28 & 3 & 11 & 25 & 89 \\
\hline Subcomisionado a Comisionado & 37 & 6 & 16 & 31 & 84 \\
\hline Total general de graduados en Ascensos & 1,354 & 109 & $8 \%$ & 1,245 & $92 \%$ \\
\hline
\end{tabular}

En el período de $1^{\circ}$ de junio de 2012 al 31 de julio de 2013 se impartieron los cursos de ascenso en las categorías de: Sargento a Subinspector a un total de 179 personas, (de estas el 25 de julio se graduaron 33 personas); Promoción Interna de Agentes y Cabos a Subinspector a un total de 144 personas, y en el curso de Inspector Jefe a Subcomisionado a un total de 64 personas (de estas el 14 de junio se graduaron 28 personas).

Cursos de Actualización, Especialidades y Filosofía en Policía Comunitaria: La Academia Nacional de Seguridad Pública entre el 01 de junio de 2009 al 31 de julio de 2013 ha capacitado un total de 16,892 integrantes de la PNC en Cursos de Actualización en Puestos de Trabajo, lográndose también la especialización de un total de 7,739 integrantes de la PNC; adicionalmente se formaron 17,882 policías en cursos de Filosofía en Policía Comunitaria, para un total de $\mathbf{4 2 , 5 1 3}$ policías formados en 1786 cursos; de estos en el período de 01 de junio de 2012 al 31 de julio de 2013 se ha capacitado a 5,475 integrantes de la PNC en 237 Cursos de Actualización; 2,745 en 89 Cursos de Especialización y 7,051 en 353 cursos de Filosofía en Policía Comunitaria. En total se capacitaron en este período un total de 15,271 en 679 cursos.

\begin{tabular}{|c|c|c|c|c|c|c|}
\hline \multicolumn{7}{|c|}{$\begin{array}{l}\text { CURSOS DE ACTUALIZACIÓN, ESPECIALIDADES Y FILOSOFÍA EN POLICÍA COMUNITARIA } \\
\text { junio } 2009 \text { a julio } 2013\end{array}$} \\
\hline Cursos & $\begin{array}{l}\text { Cantidad de } \\
\text { cursos }\end{array}$ & $\begin{array}{c}\text { Cantidad de integrantes de } \\
\text { la PNC }\end{array}$ & Mujeres & $\%$ & Hombres & $\%$ \\
\hline $\begin{array}{l}\text { Actualización en puestos de } \\
\text { trabajo }\end{array}$ & 680 & 16,892 & 1,971 & $12 \%$ & 14,921 & $88 \%$ \\
\hline Cursos de Especialidades & 237 & 7,739 & 989 & $13 \%$ & 6,750 & $87 \%$ \\
\hline $\begin{array}{c}\text { Cursos de Filosofía en Policía } \\
\text { Comunitaria }\end{array}$ & 869 & 17,882 & 2,130 & $12 \%$ & 15,752 & $88 \%$ \\
\hline Total general de graduados & 1,786 & 42,513 & 5,090 & $13 \%$ & 37,423 & $87 \%$ \\
\hline
\end{tabular}

Cursos de capacitación de Seguridad Privada: En cumplimiento al Decreto Legislativo 226 y 227 del año 2000, la Academia Nacional de Seguridad Pública impartió 250 cursos, atendiendo una cantidad de 10,698 participantes, como se muestra en el cuadro: 


\begin{tabular}{|c|c|c|c|c|c|}
\hline \multicolumn{6}{|c|}{ CURSOS DE CAPACITACIÓN DE SEGURIDAD PRIVADA (junio de 2009 a julio de 2013) } \\
\hline Cantidad de cursos & Cantidad de integrantes & Mujeres & $\%$ & Hombres & $\%$ \\
\hline 250 & 10,698 & 638 & $6 \%$ & 10,060 & $94 \%$ \\
\hline
\end{tabular}

En el período de 01 junio de 2012 a 31 julio de 2013 se impartieron un total 67 cursos de capacitación de Seguridad Privada a un total de 2,460 personas.

En total, desde el $1^{\circ}$ de junio de 2009 al 31 de julio de 2013, la ANSP ha capacitado a $\mathbf{5 8 , 9 4 9}$ personas en Formación Inicial, Ascensos, Actualización y Especialidades, Filosofía en Policía Comunitaria; y en Seguridad Privada.

Incremento de participación de mujeres en el nivel Básico: Como resultado de las medidas tomadas para la erradicación de prácticas de discriminación y violencia de género, en marzo de 2012 se graduó la promoción 105 del Nivel básico, que fue conformada por 93 hombres (37\%) y 160 mujeres (63\%), una graduación policial sin precedentes en El Salvador y probablemente en la región latinoamericana. Asimismo, en las seis últimas promociones de la 105 a la 110 ingresaron un total de 2,466 estudiantes, de los que 1,460 , equivalentes al $59.20 \%$, son hombres y 1,006 que corresponden al $40.80 \%$ son mujeres, con lo cual la meta establecida en nuestra Política Institucional de Equidad e Igualdad de Género (PIEIG) de llegar a junio del 2014 a un $40 \%$ de participación de mujeres en las nuevas promociones, ha sido cumplida de manera anticipada y con creces. Lo anterior implica un aumento del $447 \%$ de participación de mujeres en las promociones de esta Academia, ya que en las administraciones anteriores dicha participación era apenas del $9.13 \%$, en promedio; por lo que llegar a más del $40 \%$ significa, en términos cuantitativos; un incremento histórico e inédito.

\begin{tabular}{|c|c|c|c|c|c|c|c|}
\hline \multicolumn{8}{|c|}{$\begin{array}{l}\text { INGRESOS, GRADUACIONES Y PROYECCIONES DE GRADUACIONES DESDE LA PROMOCIÓN } 105 \text { A LA } 110 \\
\text { MARZO DE } 2011 \text { A JULIO } 2013\end{array}$} \\
\hline PROMOCIÓN & $\begin{array}{l}\text { FECHA DE } \\
\text { INGRESO }\end{array}$ & TOTAL & MUJERES & $\%$ & HOMBRES & $\%$ & $\begin{array}{c}\text { FECHA DE } \\
\text { GRADUACIÓN }\end{array}$ \\
\hline \multirow[t]{2}{*}{ P-105 } & 7 y 8 -marz-11 & 273 & 177 & $65 \%$ & 96 & $35 \%$ & $22 / 03 / 2012$ \\
\hline & & & & & & & $19 / 06 / 2012$ \\
\hline \multirow[t]{2}{*}{$\mathrm{P}-106$} & 6 y 7 -jun-11 & 330 & 97 & $29 \%$ & 233 & $71 \%$ & $20 / 06 / 2012$ \\
\hline & & & & & & & $28 / 08 / 2012$ \\
\hline \multirow[t]{2}{*}{ P-107 } & 9 y 10 -ene-12 & 426 & 154 & $36 \%$ & 272 & $64 \%$ & $04 / 12 / 2012$ \\
\hline & & & & & & & $11 / 03 / 2013$ \\
\hline $\mathrm{P}-108$ & 1 y 2 -oct-12 & 551 & 198 & $36 \%$ & 353 & $64 \%$ & 18/09/2013 \\
\hline P-109 & 7 y 8 -ene-13 & 550 & 199 & $36 \%$ & 351 & $64 \%$ & 19/12/2013 \\
\hline \multirow[t]{2}{*}{$\mathrm{P}-110$} & 24 y $25-$ jun-13 & 330 & 175 & $53 \%$ & 155 & $47 \%$ & $14 / 05 / 2014$ \\
\hline & TOTAL & 2,460 & 1,000 & $41 \%$ & 1,460 & $59 \%$ & \\
\hline
\end{tabular}

\section{ADQUISICIONES Y CONTRATACIONES EN EL PERÍODO COMPRENDIDO DE $1^{\circ}$ JUNIO 2009 AL 31 DE JULIO DE 2013}

\begin{tabular}{|l|c|c|c|}
\hline \multicolumn{4}{|c|}{ RESUMEN DE CONTRATACIONES DIVERSAS Y SUS MONTOS DE JUNIO DE 2009 A JULIO DE 2013} \\
\hline \multicolumn{1}{|c|}{ FORMA DE CONTRATACIÓN } & CANTIDAD DE PROCESOS & MONTO CONTRATADO & PORCENTAJE \\
\hline LICITACIÓN PÚBLICA POR INVITACIÓN & 14 & $\$ 763,927.97$ & $6 \%$ \\
\hline LICITACIÓN PÚBLICA & 36 & $\$ 8,578,639.92$ & $64 \%$ \\
\hline CONTRATACIÓN DIRECTA & 20 & $\$ 567,648.82$ & $4 \%$ \\
\hline LIBRE GESTIÓN & 2296 & $\$ 3,508,238.75$ & $26 \%$ \\
\hline MERCADO BURSÁTIL (BOLPROS) & 3 & $\$ 80,753.00$ & $1 \%$ \\
\hline \multicolumn{2}{|c|}{ TOTAL CONTRATADO } & $\$ 13,499,208.46$ & $100 \%$ \\
\hline
\end{tabular}


Informe de rendición de cuentas ANSP, junio 2012 - julio 2013

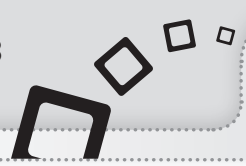

\section{GRÁFICO COMPARATIVO DE LAS DISTINTAS FORMAS DE CONTRATACIÓN Y SUS MONTOS POR EL PERÍODO COMPRENDIDO DEL 1 DE JUNIO DE 2009 \\ AL 31 DE JULIO DE 2013}

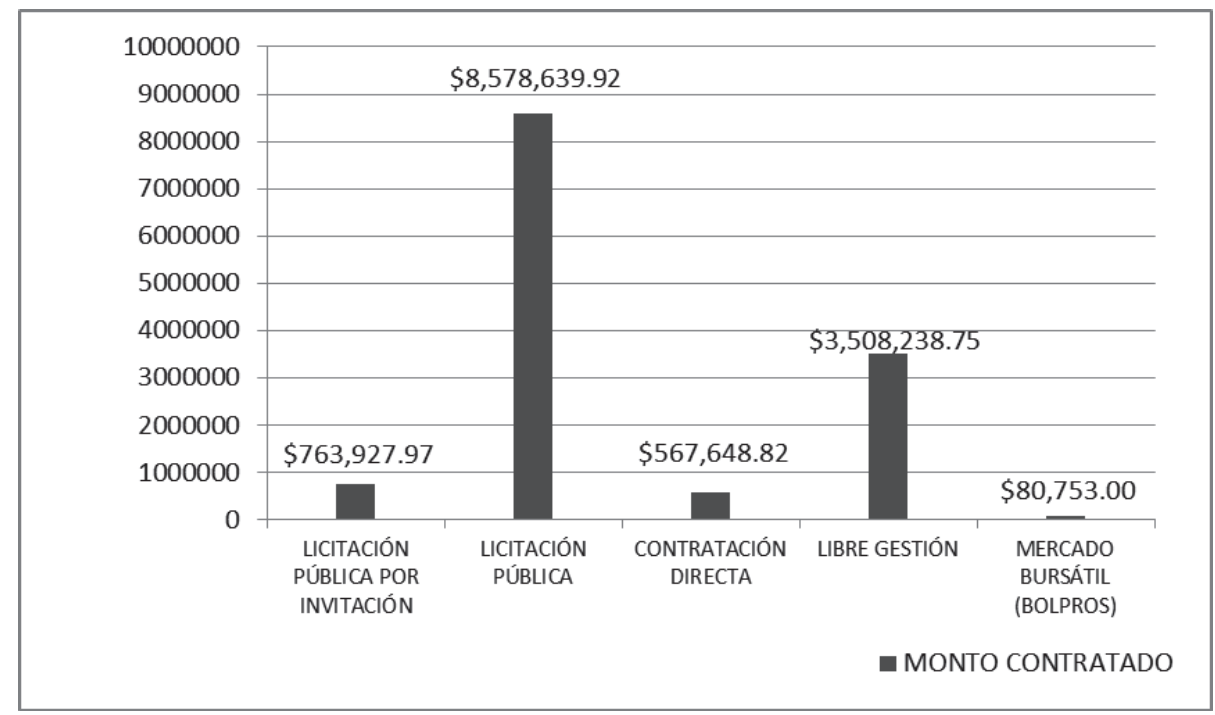

De conformidad a los montos contratados, la licitación pública y la libre gestión, fueron las formas de contratación más empleadas en la ANSP, durante el período informado.

Para el período de 01 de junio de 2012 a 31 de julio de 2013 la Academia Nacional de Seguridad Pública ha realizado las adquisiciones siguientes, detalladas en el cuadro:

\begin{tabular}{|l|c|c|c|}
\hline \multicolumn{1}{|c|}{ FORMA DE CONTRATACIÓN } & $\begin{array}{c}\text { CANTIDAD DE } \\
\text { PROCESOS }\end{array}$ & $\begin{array}{c}\text { MONTO } \\
\text { CONTRATADO }\end{array}$ & PORCENTAJE \\
\hline LICITACIÓN PÚBLICA & 16 & $\$ 3,451,977.00$ & $74.1 \%$ \\
\hline CONTRATACIÓN DIRECTA & 4 & $\$ 104,424.00$ & $2.2 \%$ \\
\hline LIBRE GESTIÓN & 629 & $\$ 1,083,013.57$ & $23.3 \%$ \\
\hline MERCADO BURSÁTIL (BOLPROS) & 1 & $\$ 17,741.40$ & $0.4 \%$ \\
\hline TOTAL & & $\$ 4,657,155.97$ & $100 \%$ \\
\hline
\end{tabular}

\section{GESTIÓN FINANCIERA Y EJECUCIÓN PRESUPUESTARIA JUNIO 2009 A JULIO DE 2013}

\begin{tabular}{|l|l|l|l|c|c|}
\hline \multicolumn{1}{|c|}{ RUBRO } & \multicolumn{1}{|c|}{ ASIGNADO } & EJECUTADO & DISPONIBLE & $\begin{array}{c}\text { EJECUTADO } \% \\
\text { POR RUBRO }\end{array}$ & $\begin{array}{c}\text { DISPONIBLE } \% \\
\text { DEL PERIODO }\end{array}$ \\
\hline 51 REMUNERACIONES & $\$ 21,868,179.26$ & $\$ 21,567,269.62$ & $\$ 300,909.64$ & $99 \%$ & $1 \%$ \\
\hline $\begin{array}{l}54 \text { BIENESY } \\
\text { SERVICIOS }\end{array}$ & $\$ 18,215,175.21$ & $\$ 16,730,936.32$ & $\$ 1,484,238.89$ & $92 \%$ & $8 \%$ \\
\hline $\begin{array}{l}55 \text { GASTOS } \\
\text { FINANCIEROS }\end{array}$ & $\$ 773,238.59$ & $\$ 725,992.75$ & $\$ 47,245.84$ & $94 \%$ & $6 \%$ \\
\hline 56 TRANSFERENCIAS & $\$ 4,227,199.07$ & $\$ 4,149,823.36$ & $\$ 77,375.71$ & $98 \%$ & $2 \%$ \\
\hline 61 ACTIVO FIJO & $\$ 2,144,143.80$ & $\$ 1,992,922.87$ & $\$ 151,220.93$ & $93 \%$ & $7 \%$ \\
\hline TOTAL EJECUTADO & $\$ 47,227,935.93$ & $\$ 45,166,944.92$ & $\$ 2,060,991.01$ & & $24 \%$ \\
\hline
\end{tabular}




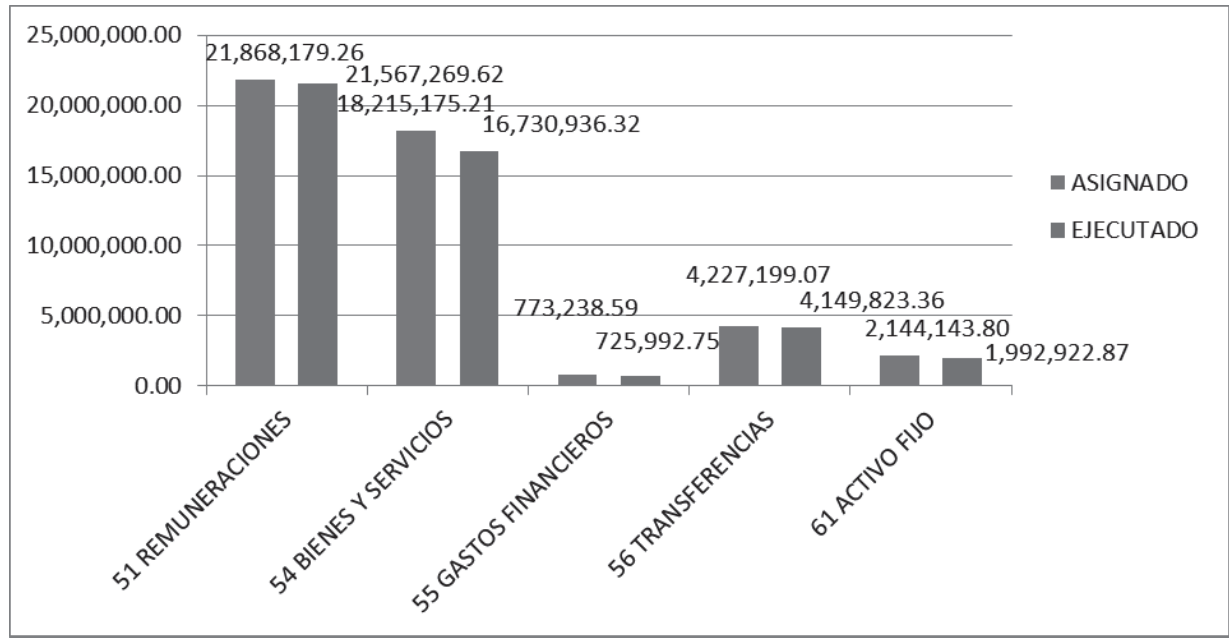

En el período de $1^{\circ}$ de junio de 2012 al 31 de julio de 2013 la Academia Nacional de Seguridad Pública a realizado la ejecución presupuestaria siguiente:

\begin{tabular}{|c|c|c|c|c|c|c|}
\hline RUBRO & ASIGNADO & EJECUTADO & & SPONIBLE & $\begin{array}{c}\% \text { DEL } \\
\text { EJECUTADO }\end{array}$ & $\begin{array}{c}\text { DISPONIBLE } \\
\text { DEL } \\
\text { PERIODO }\end{array}$ \\
\hline 51. REMUNERACIONES & $\$ 6,544,053.88$ & $\$ \quad 6,412,128.91$ & $\$$ & $131,924.97$ & $97.98 \%$ & $2.02 \%$ \\
\hline 54. BIENES Y SERVICIOS & $\$ 5,102,864.18$ & $\$ \quad 5,031,273.50$ & $\$$ & $71,590.68$ & $98.60 \%$ & $1.40 \%$ \\
\hline 55 GASTOS FINANCIEROS & $191,700.89$ & $191,700.56$ & $\$$ & 0.33 & $100 \%$ & $0 \%$ \\
\hline 56 TRANSFERENCIAS & $\$ 1,067,354.58$ & $\$ 1,067,354.03$ & $\$$ & 0.55 & $100 \%$ & $0 \%$ \\
\hline 61 ACTIVO FIJO & $872,742.38$ & $838,242.23$ & $\$$ & $34,500.15$ & $96.05 \%$ & $3.95 \%$ \\
\hline TOTAL EJECUTADO & $\$ 13,778,715.91$ & $\$ 13,540,699.23$ & $\$$ & $238,016.68$ & & $7.37 \%$ \\
\hline$\%$ EJECUTADO & & $98.27 \%$ & & $1.73 \%$ & & \\
\hline
\end{tabular}

La Academia Nacional de Seguridad Pública en el período de junio de 2009 a julio de 2013 ha recibido refuerzos presupuestarios cuyo detalle se presenta a continuación:

* Se recibió Refuerzo presupuestario por el monto de \$998,636.00 para contratación de docentes, adquisición de bienes y servicios y equipo. Aprobado el 8 de septiembre de 2010.

* Refuerzo presupuestario por el monto de $\$ 854,156.00$ del Ministerio de Justicia y Seguridad Pública, para diferentes necesidades institucionales tales como: remuneraciones, prendas de vestir, artículos de enseñanza, munición, servicio de alimentación y pago de horas clases de curso de ascenso. Aprobado el 21 de junio de 2011.

* Refuerzo presupuestario por el monto de $\$ 800,000$ del Ministerio de Justicia y Seguridad Pública, para sufragar costos del ingreso de la Promoción 108. Además se adquirió un autobús por el monto de $\$ 149,300.00$ para transporte del personal. Aprobado el 29 de junio de 2012. 
Informe de rendición de cuentas ANSP, junio 2012 - julio 2013

\section{COOPERACIÓN INTERNACIONAL}

\begin{tabular}{|c|c|}
\hline \multicolumn{2}{|l|}{ COOPERACIÓN INTERNACIONAL EN EL PERÍODO DE JUNIO 2009 A JULIO 2013} \\
\hline COOPERACIÓN DONANTE & MONTO OTORGADO \\
\hline Agencia Española de Cooperación Internacional para el Desarrollo (AECID) & $\$ 263,402.45$ \\
\hline Programa para la Aplicación de la Ley y Narcóticos (INL) & $\$ 317,710.77$ \\
\hline Agencia Japonesa de Cooperación Internacional (JICA) & $\$ 129,000$ \\
\hline Fondo de Población de las Naciones Unidas (UNFPA) & $\$ 29,293.29$ \\
\hline Fondo de las Naciones Unidas para la Infancia (UNICEF) & $\$ 29,571$ \\
\hline Justice Education Society & $\$ 118,000$ \\
\hline Plan Internacional & $\$ 4,000$ \\
\hline TOTAL & $\$ 890,978$ \\
\hline
\end{tabular}

En el período de $1^{\circ}$ de junio de 2012 al 31 de julio de 2013 se ha beneficiado a la Academia Nacional de Seguridad Pública con las siguientes cooperaciones internacionales, detalladas en el cuadro siguiente:

\begin{tabular}{|l|c|}
\hline \multicolumn{2}{|c|}{ COOPERACIÓN INTERNACIONAL EN EL PERÍODO DE JUNIO 2012 A JULIO 2013 } \\
\hline \multicolumn{1}{|c|}{ COOPERACIÓN DONANTE } & MONTO OTORGADO \\
\hline Agencia Española de Cooperación Internacional para el Desarrollo (AECID) & $\$ 15,000$ \\
\hline Embajada de Francia con sede en Costa Rica & $\$ 52,090.00$ \\
\hline Gobierno de Japón & $\$ 120,000.00$ \\
\hline Justice Education Society & $\$ 15,000$ \\
\hline Programa de las Naciones Unidas para el Desarrollo (PNUD) & $\$ 48,740.00$ \\
\hline Programa para la aplicación de la ley y narcóticos (INL) & $\$ 62,857$ \\
\hline \multicolumn{2}{|c|}{ TOTAL } \\
\hline
\end{tabular}

\section{CENTRO DE INVESTIGACIÓN CIENTÍFICA}

En cumplimiento a la Ley Orgánica de la ANSP, específicamente su artículo 3, inciso "c" que establece como atribución de esta Academia: "Investigar, estudiar y divulgar materias relativas a la Policía Nacional Civil y la Seguridad Pública", bajo la presente administración fue creado el Centro de Investigación Científica, CINC, de la ANSP en 2010; dicho centro ha realizado actividades de producción y divulgación académica a través de la revista científica "Policía y Seguridad Pública", de la que se han publicado tres números monotemáticos referidos a: "Policía Comunitaria y Prevención de Violencia", "Criminalidad Organizada Transnacional"; y "Narcotráfico y Lavado de Activos"; actualmente se prepara el número cuatro que abordará la temática de "Violencia Carcelaria y Población Reclusa" y será presentado en el mes de agosto. La revista está apoyada en un sistema de arbitraje en el que han participado más de 30 especialistas de distinta filiación académica y laboral entre institucionales, nacionales e internacionales así como también de las más distintas formaciones disciplinarias. Su distribución internacional incluye el despacho a más de 123 destinos entre universidades, centros de pensamiento, redes de expertos y centros de investigación. Asimismo el Centro de Investigación Científica, apoyado en la cooperación internacional inauguró y puso en funcionamiento un Centro de Documentación e Información más Biblioteca Virtual especializados para investigadores y estudiantes de la ANSP de los niveles superiores, el cual a partir del 29 de mayo ha puesto a disposición más de 500 referencias bibliográficas físicas y más de 6,000 referencias virtuales, entre ellas una colección especial de notas periodísticas de cuatro periódicos nacionales de mayor circulación, correspondientes al área de seguridad pública de los años 1992 y 1993. 
Además, a partir del 19 de junio del presente año la revista "Policía y Seguridad Pública" ingresó al catálogo Latindex, un gestor web del Sistema Regional de información en línea para Revistas Científicas de América Latina, el Caribe, España y Portugal que tiene sede en México; cumpliendo 30 parámetros de calidad y gestión editorial de un total de 33 establecidos. Convirtiéndose en la primera publicación indexada del sector público, la sexta a nivel nacional y la primera de la ANSP; condición que contribuirá a la generación de mayor valor agregado e impulso a los esfuerzos institucionales para la acreditación de esta entidad como Instituto de Educación Superior (IES).

\section{UNIDAD DE ACCESO A LA INFORMACIÓN PÚBLICA INSTITUCIONAL (UAIP)}

Con el objetivo de dar cumplimiento a la Ley de Acceso a la Información Pública, cuyo objetivo es garantizar el acceso de toda persona a la información pública generada, administrada o en poder de las instituciones del Estado, se creó por medio del acuerdo DGE-A-037-2011 la Unidad de Acceso a la Información Pública Institucional de la ANSP y posteriormente, para los efectos de proveer un mejor el servicio al ciudadano se creó también la Oficina de Información y Respuesta en el mes de agosto de 2011; convirtiéndose de esta manera en el principal mecanismo de contribución de fomento de la transparencia de las actuaciones de la ANSP.

La ANSP a través de su Unidad de Acceso a la Información Pública Institucional ha permanecido en el primer lugar del ranking de publicación de sitios web de la Sub Secretaría de Transparencia y Anticorrupción entre un total de 68 instituciones del Órgano Ejecutivo y 5 municipalidades. Además, en coordinación con la Universidad Modular Abierta en octubre/noviembre de 2012 se impartió el "Diplomado en Gestión de Unidades de Información con enfoque Archivístico", graduando a un total 47 personas del área administrativa de la institución. Asimismo en diciembre de 2012 y mayo de 2013 la Oficina de Información y Respuesta participó en 2 ferias de transparencia organizadas por la Secretaria de Asuntos Estratégicos de la Presidencia de la República a través de su Subsecretaría de Transparencia y Anticorrupción.

\section{POLÍTICA INSTITUCIONAL DE EQUIDAD E IGUALDAD DE GÉNERO, PIEIG}

La Dirección General de la Academia, ha emitido y aplicado la circular 008/2009 que contiene las "Instrucciones contra el acoso sexual y otras formas de violencia de género especialmente contra las mujeres" y la circular 001/2011 que contiene las "Directrices contra prácticas discriminatorias". En noviembre de 2011 fue aprobada y socializada la Política Institucional de Equidad e Igualdad de Género (PIEIG), para dar respuesta efectiva a la problemática de género en la Academia, esta tiene como propósito fomentar en el comportamiento de las autoridades, jefaturas, personal docente, administrativo, policial en comisión de servicio y alumnado, relaciones de igualdad y de no discriminación por motivos de género desde un enfoque de derechos humanos. Este cambio de paradigma ya está teniendo efecto y se refleja en los procesos, procedimientos y decisiones institucionales. Asimismo, para dar cumplimiento a la Ley Especial Integral para una Vida Libre de Violencia para las Mujeres, así como al Instructivo sobre Relaciones Laborales en el Órgano Ejecutivo, en agosto de 2012 la Dirección General de la ANSP emitió el "Instructivo para la Detección, Atención, Protección de Víctimas, Investigación y Sanción de la Violencia de Género, Acoso Laboral y Sexual", basado en la normativa antes citada. En diciembre de 2012, mediante Acuerdo DGE-A.053/2012, se crea la Unidad de Género Institucional que tiene como atribución principal formular y coordinar la implementación de la PIEIG, así como planificar, facilitar e implementar su Plan de Acción. 


\section{MAYOR RESPETO Y FORTALECIMIENTO DE LOS DERECHOS DE LOS TRABAJADORES Y TRABAJADORAS.}

Siguiendo los lineamientos del Presidente de la República, de favorecer los derechos de las trabajadoras y trabajadores del sector público, bajo la presente administración se ha garantizado la estabilidad del personal de la ANSP. En estos cuatro años apenas fueron despedidos el equivalente al $4 \%$ del total del personal. Asimismo, fue organizado y autorizado por el Ministerio de Trabajo, el primer sindicato de la institución, denominado: Sindicato de Trabajadores y Trabajadoras de la ANSP, SITANSP, con el cual se realizó un proceso de negociación que en julio de 2011 dio como resultado la suscripción del primer Contrato Colectivo de Trabajo, el cual entró en vigencia después de su inscripción en el Ministerio de Trabajo el día 5 de julio de 2013. Otro logro reciente, como resultado de las gestiones realizadas por la Dirección General de la Academia, fue la autorización del Ministerio de Hacienda para que a partir del primero de enero de 2013, los trabajadores y trabajadoras que ocupaban plazas por contrato, pasaran al sistema de Ley de Salarios, con lo cual todos los trabajadores permanentes de la ANSP gozarán de mayor estabilidad y otras garantías laborales. Por primera vez en 20 años de fundación de la ANSP, se celebró el primer contrato colectivo de trabajo entre la Academia Nacional de Seguridad Pública y el Sindicato de trabajadores y trabajadoras de la ANSP, SINTANSP, el día 5 de julio de 2013 se inscribió en el Departamento de Organizaciones Sociales adscrito a la Dirección General de Trabajo dicho contrato, con este requisito se aplicará el mismo garantizando y haciendo irreversibles los derechos, prestaciones y beneficios laborales de todos los trabajadoras y trabajadores. Asimismo, se ha mantenido un proceso de diálogo permanente con representantes del SITANSP para tratar puntos de interés tanto de los trabajadores como de la administración. Se ha conformado la Mesa de relaciones laborales para mejorar el entendimiento entre el SITANSP y la ANSP. La Academia también ha pasado a formar parte de la Red de Relaciones Laborales, bajo el impulso de la Secretaría para Asuntos Estratégicos de la Presidencia de la República. Además se ha tomado muy en serio la elaboración de los informes sobre relaciones laborales que deben ser presentados a dicha Secretaría. También se está trabajando en reformar el Reglamento Interno de Trabajo a fin de mejorar las condiciones laborales. Se han creado dos Comités de Seguridad e Higiene Ocupacional los que trabajan por garantizar que los empleados y empleadas cuenten con un mejor ambiente de trabajo y se minimicen los riesgos ocupacionales, con la participación del Sindicato en dichos comités.

\section{GESTIÓN EFECTIVA Y TRANSPARENTE DE LOS RECURSOS.}

Durante los cuatro años de la presente administración, la ejecución presupuestaria de la ANSP al final de cada período ha sido de $98.00 \%$, en promedio, y en cada ejercicio el techo presupuestario se ha quedado corto con respecto a las necesidades de funcionamiento y a la capacidad de ejecución; gracias al apoyo de las autoridades superiores, la Academia siempre ha obtenido los refuerzos presupuestarios que necesita para el cumplimiento de sus metas y objetivos y los ha ejecutado en un $100 \%$. Los dos logros más recientes en esta línea es que el Ministerio de Justicia otorgó una transferencia de su propio presupuesto para la ANSP por US\$ $800,000.00$, autorizada por el Ministerio de Hacienda, con lo cual se aseguró el ingreso de la promoción 108 conformada por 551 alumnos y alumnas, a inicios de octubre de 2012. El otro gran logro es que para el año 2013 se obtuvo un incremento del techo presupuestario en 1.3 millones de dólares; de este modo la ANSP alcanzó la mayor asignación presupuestaria de su historia 
por un total de US\$12.142,505.00, con lo que, entre otras cosas, se garantizó la continuidad de la promoción 108 conformada por 551 alumnos y alumnas, el ingreso de la promoción 109 en enero de 2013 conformada por 550 alumnos y alumnas; más el ingreso a finales de junio de este año de la promoción 110 integrada por 330 alumnos que se graduará en mayo de 2014 y será la última de la presente administración. Con estas tres promociones se cumplirá la meta de contar con 23,400 policías en la plantilla policial en mayo de 2014 , y se llegará a tener uno de los encuadramientos policiales más altos de América Latina pues será una tasa de 3,8 policías por cada 1,000 habitantes. En esta línea del uso eficiente y transparente de los recursos públicos, una de las mayores satisfacciones es que a más de 4 años de la presente gestión, en el rubro de boletos aéreos por misiones oficiales fuera del país, bajo esta administración no se ha comprado ni un solo boleto aéreo para cumplir ese tipo de misiones, ya que la política que se mantiene es que, siendo ésta una institución académica, si el titular o algún otro funcionario o empleado sale a cumplir una misión oficial, es por invitación de algún gobierno extranjero u otra institución internacional quienes por regla general cubren los costos de boletos aéreos, alojamientos y demás costos de los eventos académicos a los que se acude.

\section{APROBACIÓN DE AUDITORÍAS DE LA CORTE DE CUENTAS DE LA REPÚBLICA.}

En la línea de una gestión honesta y transparente, con mucha honra y satisfacción, la administración actual superó los hallazgos de auditorías de Corte de Cuentas que habían dejado las administraciones anteriores en los períodos 2007 y 2008 , y entre 2011 y lo que va del año 2012, la mencionada entidad contralora estatal realizó auditorías financieras a la ANSP de los ejercicios 2009, 2010 y 2011, de las cuales emitió los informes finales, con un dictamen limpio y cuyos resultados demuestran que la presente administración ha actuado con apego a la legalidad y transparencia en la administración de los recursos asignados. Dichos informes destacan los aspectos siguientes: a) Financieros, de los cuales se informa que los resultados de auditoría no revelan aspectos financieros a ser reportados; b) Control Interno, reportan que los resultados de las pruebas de auditoría no revelan aspectos que puedan ser reportados en el informe final y que las deficiencias de control interno determinadas, por no ser relevantes fueron presentadas en Carta de Gerencia; c) Cumplimiento de la legalidad, revelan leves deficiencias que pueden ser superadas al observar las recomendaciones de las auditorías. 